\title{
Performance of Visible Light Communications with Dimming Controls
}

\author{
Zi Feng*, George Papageorgiou*, Qian Gao ${ }^{\dagger}$, Ahmed F. Atya*, Srikanth V. Krishnamurthy*, Gang Chen ${ }^{\dagger}$ \\ ${ }^{*} \mathrm{UC}$ Riverside \{zfeng, gpapag, afath001, krish\}@cs.ucr.edu, ${ }^{\dagger} \mathrm{UC}$ Riverside \{qgao, gachen $\} @ e e . u c r . e d u$,
}

\begin{abstract}
Visible light communications (VLC) has recently gained popularity as an alternative to RF. However, the design and deployment of a VLC system requires an understanding of the underlying communications and how they affect the design of higher layer protocols. In this paper, we take a basic step towards getting an understanding of the impact of interference on a VLC system. Such an understanding is key to the design of MAC protocols for arbitrating access across lights in multiple rooms, while ensuring that illumination requirements are met. Specifically, we consider the interference across two rooms from VLC emitters. The emitters are assumed to use Binary Pulse Position Modulation (BPPM); the pulse width is varied to provide different dimming levels. In this setting, we use a modified ray-tracing algorithm to calculate the channel impulse response between the emitters and receivers that are located at different positions within a room. Subsequently, we analyze the performance observed at the receivers in the presence of (i) illumination and (ii) transmissions from an interfering VLC emitter. We find that in the former case, the VLC emissions from the interferer do not impact the reception at the target receiver. However, in the latter case, the performance is degraded. The extent of degradation depends on the position of the receiver. We find that increasing the dimming level increases the pulse intensity and thus, improves performance in the presence of interference. We also perform extensive simulations to provide performance results in different settings.
\end{abstract}

\section{INTRODUCTION}

Visible Light Communications (VLC) is gaining popularity ever since the first VLC system (utilizing white LED light) was proposed in [1]. VLC is considered to be a promising alternative to RF in indoor settings. In a VLC system, the LED lights not only illuminate a room, but can also support optical wireless communication. Currently, IEEE has a standard [2] for VLC. White light LEDs have the advantages of reliability, security, lower power consumption, easy maintenance, and cost-effeciency. They are also harmless to the human eye. Furthermore, it could be potentially easy to deploy a VLC network, since in most cases of interest (indoors) the lighting infrastructure already exists.

In [3], the authors provide an indoor VLC system design with theoretical analysis and experimental proof of the feasibility of VLC. In [4], a typical basic configuration is provided and the performance that can be achieved with different modulation schemes is discussed. Regarding the VLC channel, a simulation based method in characterizing the infrared (IR) channel that has been proposed in [5] has been broadly adopted. Based on this work, [6] presents the VLC channel characteristics considering wavelength and spectral reflectance.

Unfortunately, the above efforts do not provide an understanding of interference between VLC emissions. To

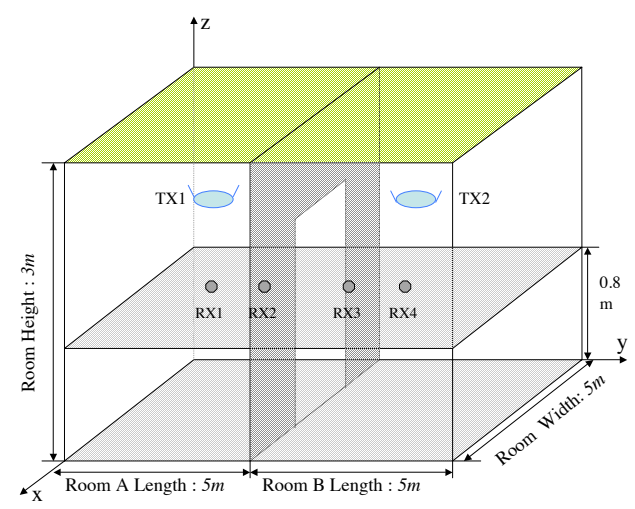

Fig. 1: The visible light system deployed across two rooms with an open door in between.

illustrate, we consider a VLC system deployed over two rooms separated by a door, with each room containing a set of emitters and receivers, as shown in Fig. 1. This type of indoor setting is typical, especially in home or office establishments. Needless to say, if the door is closed, the VLC system can provide a separate channel for each room since the visible light signal cannot go through opaque surfaces. On the other hand, if the door is open, the visible light signal in one room interferes with the signal in the next room. Understanding the impact of this interference is critical for the design of higher layer protocols for VLC, which in turn are key in making wide spread deployment of VLC a reality.

In this paper, we study the VLC communications in the presence of interference in the simple scenario shown above (Fig. 1). Although simple, the set up provides a set of key insights that can help in the design of protocols going forward. Since the primary use of the LED emitters is illumination, dimming control is one of the desired functions of the system. Thus, throughout this work, we use the Variable Pulse Position Modulation (VPPM) scheme, which combines Binary Pulse Position Modulation (BPPM) for data transmission and Pulse Width Modulation (PWM) for dimming control. Note that VPPM is easy to implement and has been discussed in [2].

Our contributions in brief: We characterize the channel based on a novel algorithm that uses a modified ray-tracing model to calculate the channel impulse response. We study the communication channel between Tx1 (see Fig. 1 and a receiver in the same room (Room 1), treating the transmission from Tx2 as interference. We utilize a simple symbol detection method and compute the Signal to Noise Ration (SNR) to characterize the quality of the connection. We use simulations to determine the SNR distribution in the room for two different cases: (a) 
TABLE I: System parameters.

\begin{tabular}{|c|c|c|}
\hline \multicolumn{2}{|l|}{ Room Length $\mathrm{x}(\mathrm{m})$} & 5 \\
\hline \multicolumn{2}{|l|}{ Room Width y(m) } & 5 \\
\hline \multicolumn{2}{|l|}{ Room Height $\mathrm{z}(\mathrm{m})$} & 3 \\
\hline \multicolumn{2}{|l|}{ Roof reflectivity } & 0.38 \\
\hline \multicolumn{2}{|l|}{ Floor reflectivity } & 0.6 \\
\hline \multicolumn{2}{|l|}{ Walls reflectivity } & 0.68 \\
\hline \multicolumn{2}{|l|}{ Door Width x(m) } & 3 \\
\hline \multicolumn{2}{|l|}{ Door Height $\mathrm{z}(\mathrm{m})$} & 2 \\
\hline \multicolumn{2}{|l|}{ Tx1 Position } & $(2.5,2.5,2)$ \\
\hline \multicolumn{2}{|l|}{ Tx2 Position } & $(2.5,7.5,2)$ \\
\hline \multicolumn{2}{|l|}{ Rx1 Position } & $(2.5,2.5,0.8)$ \\
\hline \multicolumn{2}{|l|}{ Rx2 Position } & $(2.5,4.0,0.8)$ \\
\hline \multicolumn{2}{|l|}{ Rx3 Position } & $(2.5,6.0,0.8)$ \\
\hline \multicolumn{2}{|l|}{ Rx4 Position } & $(2.5,7.5,0.8)$ \\
\hline \multicolumn{2}{|l|}{ Receiver Area $\left(\mathrm{cm}^{2}\right)$} & 1 \\
\hline \multicolumn{2}{|l|}{ Receiver FOV (deg) } & 85 \\
\hline \multirow{2}{*}{ Emitter Orientation } & $\varphi$ & 0 \\
\hline & $\theta$ & 90 \\
\hline \multirow{2}{*}{ Receiver Orientation } & $\varphi$ & 0 \\
\hline & $\theta$ & 90 \\
\hline
\end{tabular}

both emitters are transmitting, and (b) Tx1 is transmitting and $\mathrm{Tx} 2$ is illuminating.

We also provide a BER performance analysis for the system which can account for variable data rates, dimming levels and door sizes. The results show that increasing the data rate or increasing the door size can degrade the BER performance. Increasing the dimming level of $\mathrm{Tx} 1$ can improve the BER performance ${ }^{1}$ On the other hand, increasing the dimming level of the interfering emitter impacts the BER performance in a negative way, especially for the receivers close to the door.

Finally, we look at the performance when Tx 1 reduces its data rate to below that of Tx2. Our results show that this strategy can improve the BER performance significantly.

\section{A Visible Light Communication System Model}

We consider a visible light indoor optical wireless system in two rooms with an open door between them, as shown in Fig. 1. There are two LED emitters, Tx1 and Tx2, in Room 1 and Room 2, respectively. We assume that the two emitters can transmit data and illuminate at the same time. The receivers are located on a plane that is $0.8 \mathrm{~m}$ above the floor. We consider the receivers Rx1 and Rx2 that are located in Room 1 and are associated with emitter Tx1. Due to geometrical symmetry, we expect that the performance of the receivers $\mathrm{Rx} 3$ and $\mathrm{Rx} 4$, which are associated with emitter $\mathrm{T} \times 2$, to be similar to that of $\mathrm{Rx} 1$ and Rx2, respectively. The parameters of the system are shown in Table. I.

\section{A. VPPM Transmitter}

The transmitters Tx 1 and Tx2 of Fig. 1 are at a distance of $0.5 \mathrm{~m}$ from the ceiling and point straight up. We assume that each LED emitter uses VPPM modulation [2] and adopts the emission profile in [7]. The VPPM scheme is identical to the 2 -PPM scheme when the duty cycle is $50 \%$. The duty cycle $\delta$, i.e. the pulse width within the slot $T$, corresponds to the

\footnotetext{
${ }^{1} \mathrm{We}$ find that this is because, the power intensity of a pulse increases with the width.
}

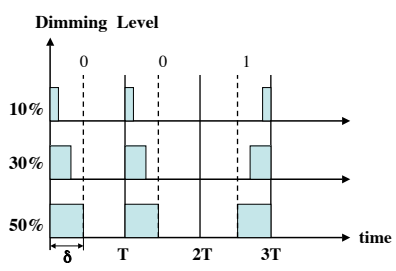

Fig. 2: An example of VPPM signals.

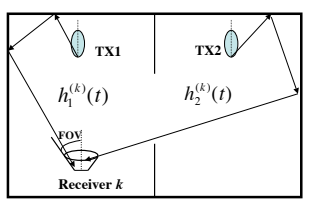

Fig. 3: Reflection of rays.

dimming level ${ }^{2}$. In this work, we consider the dimming level to be no more than $50 \%$ since we use BPPM (the pulse can occupy at most one half of the slot width). Fig. 2 provides a simple example of the VPPM signal. The $n^{\text {th }}$ transmitted bit $s_{n} \in\{0,1\}$ corresponds to the symbol $S_{n}^{\delta}$, given by:

$$
\begin{aligned}
& S_{0}^{\delta}(t)= \begin{cases}2 P_{t}, & \text { if } 0 \leq t<\delta T \\
0, & \text { otherwise }\end{cases} \\
& S_{1}^{\delta}(t)= \begin{cases}2 P_{t}, & \text { if }(1-\delta) T \leq t<T \\
0, & \text { otherwise }\end{cases}
\end{aligned}
$$

where $T$ is the duration of a VPPM symbol and $P_{t}$ is the average transmitted power over the entire time slot.

\section{B. Channel Impulse Response}

The channel impulse response for the case where the emitters and the receivers reside in the same room has been presented in [8]-[10]. We extend this previous work by considering a situation where two emitters are located in two neighboring rooms with an open door in between. It is hard to calculate analytically the channel path loss in this case due to interference. Instead and motivated by [10], we propose the use of a modified ray-tracing algorithm to generate the channel impulse response $h_{i}^{(k)}(t)$ for the channel between emitter $i$ and receiver $k$. For a receiver $k$ in Room 1, we consider two separate channels: (i) the channel between emitter Tx1 and the receiver $h_{1}^{(k)}(t)$, (ii) the channel between emitter Tx 2 and the receiver $h_{2}^{(k)}(t)$.

We calculate the impulse response $h_{i}^{(k)}(t)$ by means of simulation that lasts $t_{-} \max$ sec. The simulator determines the maximum number ray_max of rays to generate. The selection of the number of rays is discussed in [11]. The distribution of the generated rays is according to the emission profile. The propagation path of each ray may contain obstacles. These obstacles include the roof, the ceiling and the walls. Note that we assume the door is open, so the door is not considered an obstacle.

When a ray reaches an obstacle, the simulator checks if the point of impact $(P I)$ is on the door. If the $P I$ is not on the door, it reflects the ray and the power is reduced by the reflection coefficient of the obstacle. Subsequently, a new ray is generated at $P I$ with the new, reduced, power. If the $P I$ is on the door, the ray propagates to the other room. The simulator computes the new point of impact $P I^{\prime}$ in the other room and generates a new ray at that point. In Fig. 3 the reflections of

\footnotetext{
${ }^{2}$ Note that when we say the dimming level increases, we mean that the pulse width increases. This also translates to a higher average power.
} 
while $r a y \_n u m<r a y \_m a x$ do

step 1: Generate a new ray starting at the emitter ;

$t=0, P=1$;

step 2 : while $t<t \_\max$ do

Propagate the ray until it reaches any obstacle

plane;

Find the point of impact $P I$ where the ray

intersects with the obstacle;

if PI is on the door then

Propagate the ray to the neighboring room;

Find the impact point $P I^{\prime}$ where the ray

intersects with any obstacle planes in the neighbor room;

Calculate the contribution from $P I^{\prime}$ to the receiver;

Generate a new ray starting at $P I^{\prime}$, with

reduced power $P=\rho P$;

Back to Step 2;

else

Calculate the contribution from $P I$ to the

receiver;

Generate a new ray starting at $P I$;

end

end

end

Increase ray_numby 1 .

Algorithm 1: Ray-tracing Algorithm for $h_{i}^{(k)}(t)$

the rays are shown. Only diffused reflection is considered in this algorithm. The direct power contribution of each ray is calculated each time it is reflected [10]. The calculated power is added to $h_{i}^{(k)}(t)$ if the ray can be intercepted by the receiver, i.e. it is within the FOV of the receiver.

Fig. 4 presents the impulse response computed using Algorithm 1 for the channel between Tx 1 and receivers Rx 1 and $\mathrm{Rx} 2$. The number of rays is 10,000 , the resolution time is $0.2 \mathrm{~ns}$ and the simulation time is $120 \mathrm{~ns}$. The shapes of $h_{1}^{(1)}(t)$ and $h_{1}^{(2)}(t)$ look similar. Only the peak power of $h_{1}^{(1)}(t)$ is higher than $h_{1}^{(2)}(t)$. This is due to the positions of $\mathrm{Rx} 1$ and $\mathrm{Rx} 2$, i.e., $\mathrm{Rx} 1$ is closer to $\mathrm{Tx} 1$ and further from $\mathrm{Tx} 2$ while $\mathrm{Rx} 2$ is further to Tx1 and closer to Tx2. For the same reason, we see that the power of $h_{2}^{(1)}(t)$ is much smaller than the power of $h_{2}^{(2)}(t)$.

\section{Received Signal}

Receivers in Room 1 are associated with Tx 1 and receivers in Room 2 are associated with Tx2. If not explicitly stated otherwise, we assume that the emitters Tx1 and Tx2 use the same data rate and the system is synchronized. Looking at the performance in Room 1, we consider two scenarios: (i) both $\mathrm{Tx} 1$ and $\mathrm{Tx} 2$ are transmitting data, and (ii) Tx1 is transmitting data and $\mathrm{T} \times 2$ is just illuminating.

Receivers $\mathrm{Rx} 1$ and $\mathrm{Rx} 2$ are connected to $\mathrm{Tx} 1$, therefore the signal from Tx2 acts as an interference to them. Following [5], the received signal $r_{1}^{k}(t)$ at receiver $k$ in Room 1 is given as:

$$
r^{(k)}(t)=R^{(k)} X_{1}(t) \otimes h_{1}^{(k)}(t)+I^{(k)}(t)+n(t)
$$
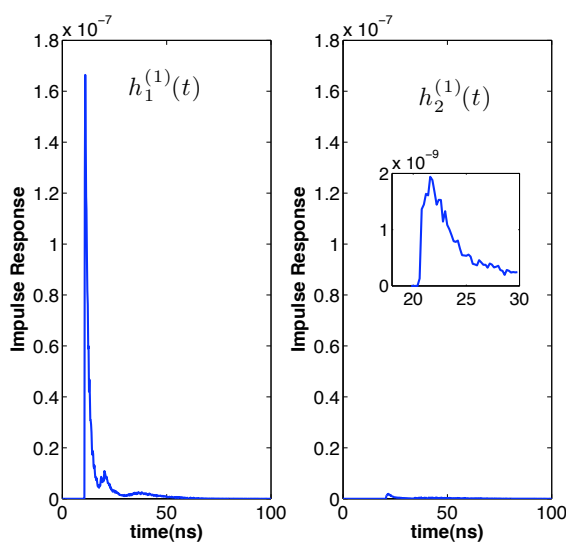

(a) Impulse Response between transmitters and Rx1.
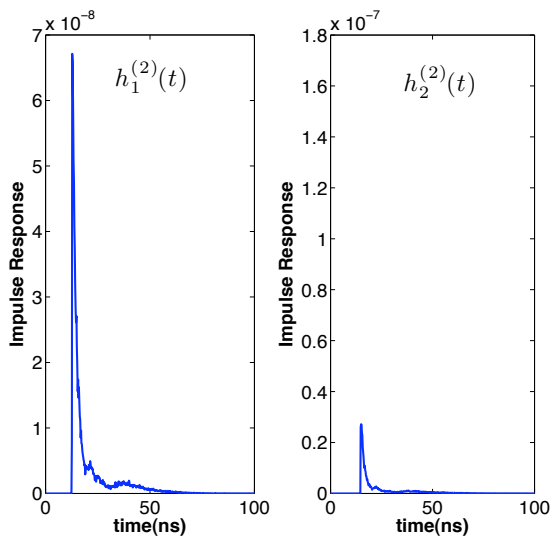

(b) Impulse Response between transmitters and $\mathrm{Rx} 2$

Fig. 4: Impulse Responses.

where $R^{k}$ is the responsivity of receiver $\mathrm{k}$ in Room $1, X_{1}(t)$ is the transmitted signal of Tx 1 and $n(t)$ is the noise. $I^{(k)}(t)$ is the interference from $\mathrm{Tx} 2$ to receiver $k$, given by:

$$
I^{k}(t)=R^{(k)} X_{2}(t) \otimes h_{2}^{(k)}(t)
$$

where $X_{2}(t)$ is the transmitted signal of $\mathrm{Tx} 2$. Note that for scenario (ii) above $X_{2}(t)$ is a signal with constant power. We further discuss this case in Section II-D. The variance $\sigma_{\text {total }}^{2}$ of the Gaussian $n(t)$ [1], [3], [5] is given by:

$$
\sigma_{\text {total }}^{2}=\sigma_{\text {thermal }}^{2}+\sigma_{\text {shot }}^{2}
$$

The shot noise variance is given by:

$$
\sigma_{\text {shot }}^{2}=2 q R P_{n} I_{2} R_{b}
$$

where $q$ is the electric charge, $R$ is the photodiode responsivity, $P_{n}$ is the noise power, $I_{2}$ is the noise bandwidth factor and $R_{b}$ is the data rate. The thermal noise variance is given by:

$$
\begin{aligned}
\sigma_{\text {thermal }}^{2}= & \frac{4 k T_{f}}{R_{F}} I_{2} R_{b}+\frac{16 \phi^{2} k T_{f}}{g_{m}}\left(\Gamma+\frac{1}{g_{m} R_{D}}\right) C_{T}^{2} I_{3} R_{B}^{3} \\
& +\frac{4 \phi^{2} K I_{D}^{a} C_{T}^{2}}{g_{m}^{2}} I_{f} R_{b}^{2}
\end{aligned}
$$

We adopt the parameters defined in [5] except for the data rate $R_{b}$. The received waveform can be calculated using (3)-(7). 


\section{Symbol Detection and SNR Distribution}

There are various methods designed for symbol detection [12]-[14]. We need a symbol detection mechanism that is simple and effective. As discussed earlier, the system that we consider in this work has two channel impulse responses $h_{1}(t)$ and $h_{2}(t)$ for each receiver. Note that these two channel impulse responses are different and independent of each other; $X_{1}(t)$ and $X_{2}(t)$ are also different, i.e. Tx1 and Tx2 are transmitting different data. Thus, equalization [15] cannot be employed in this system. Considering BPPM where the pulse is confined to half a slot (i.e., $\delta \leq 0.5$ ) we can neglect ISI (Intersymbol Interference) when the slot period is sufficiently longer than the delay spread.

The non-equalized receiver performs symbol-by-symbol ML detection. We assume that the receiver is synchronized with the transmitter and the receiver has the information of dimming level and the data rate of the transmitter it is associated with, prior to the data transmission [2]. Thus the received signal $r(t)$ can be sampled into two blocks $y_{1}, y_{2}$ for each symbol, where $y_{1}$ and $y_{2}$ correspond to the samples in the fist half slot slot $_{1}$ and second half slot slot $_{2}$ respectively. The receiver makes symbol decisions based on the relative magnitude of $y_{1}, y_{2}$.

Using this symbol detection method, the SNR can be defined as:

$$
\mathrm{SNR}_{s_{n}}=\frac{\left|P_{\text {slot }_{1}}-P_{\text {slot }_{2}}\right|}{P_{\text {noise }}},
$$

where $P_{\text {noise }}=\sigma_{\text {total }}$ (see (5)), $s_{n}$ is the desired symbol and $P_{\text {slot }}$ and $P_{\text {slot }}$ are the average received power levels in the first half slot slot $_{1}$ and the second half slot slot $_{2}$, respectively. The average received power is computed based on the received signal $r^{(k)}(t)$, which can be computed by (3) We are interested in the expected value of the SNR across Room 1, which provides a measure of the communication performance in Room 1. As discussed earlier, we consider two scenarios: (i) both $\mathrm{Tx} 1$ and $\mathrm{T} \times 2$ are transmitting, and (ii) $\mathrm{Tx} 1$ is transmitting and Tx2 is just illuminating. Assuming the input bit stream is an independently and identically distributed (i.i.d.) Bernoulli(1/2) process, the emitter in transmission mode transmits a symbol ${ }^{3} 0$ or 1 with probability $1 / 2$.

Considering the first scenario, the possible combination of symbols from $\mathrm{Tx} 1$ and $\mathrm{Tx} 2$ is one in the set $S^{(1)}=$ $\{(0,0),(0,1),(1,0),(1,1)\}$. The expected value of the SNR for the first scenario is:

$$
\mathrm{E}[\mathrm{SNR}]=\frac{1}{4} \sum \mathrm{SNR}_{\left\{s_{1}, s_{2}\right\}}
$$

where $\left\{s_{1}, s_{2}\right\} \in S^{(1)}$ and $s_{1} s_{2}$ are the bits transmitted from Tx1 and Tx2 respectively. To compute $P_{\text {slot }}$ and $P_{\text {slot }}$ for a specific receiver $k$ in Room 1, we first simulate its $h_{1}^{(k)}(t)$ and $h_{2}^{(k)}(t)$ with Algorithm 1. $\mathrm{SNR}_{s_{1}, s_{2}}$ is computed using (8) and $s_{1}$ is considered to be the desired symbol, we look at receivers in Room 1.

Regarding the second scenario, $\mathrm{T} \times 2$ is in illumination mode so that the signal $S_{\text {illumin }}$ it generates is of constant power:

$$
S_{\text {illumin }}^{\delta}(t)=2 \delta P_{t}
$$

\footnotetext{
${ }^{3}$ To evaluate the performance of the system, we consider the system is uncoded throughout this work.
}

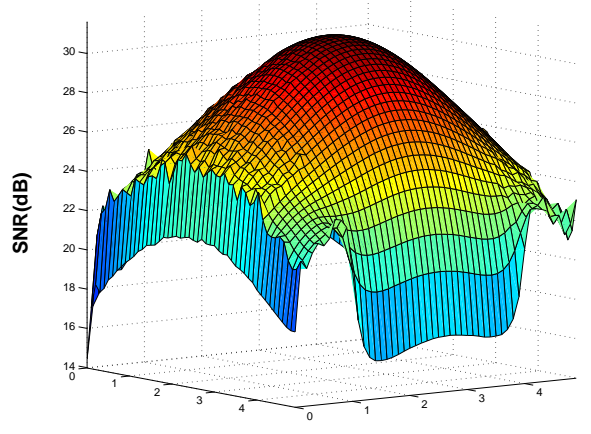

(a) Both Tx1 and Tx2 are transmitting.



(b) Only Tx1 is transmitting.

Fig. 5: SNR distribution of Room 1 (datarate $1 \mathrm{Mb} / \mathrm{s}$ )

where $\delta$ is the dimming level of Tx2. Fig. 5 shows the expected value of the SNR across Room 1 for scenario (i) and (ii). The dimming level of both emitters is 0.5 and the data rate of the communicating transmitter is $1 \mathrm{Mbps}$. The receiver plane is at a distance of $0.8 \mathrm{~m}$ above the floor. Comparing the results shown in Fig. 5(a) and Fig. 5(b), we observe that if Tx2 is just illuminating it does not impact the transmission performance in Room 1, while if both Tx1 and Tx2 are transmitting data, the performance in Room 1 is affected. The effect is especially harsh for the receivers in Room 1 closer to the door; for these the SNR is degraded largely.

\section{BER PERFORMANCE}

In this section we first provide basic BER performance analysis and then look into the performance with different system parameters. Our goal is to achieve an optimal BER performance by tuning the dimming level and the data rate for the system we consider in this work.

\section{A. Bit Error Rate Analysis}

First we seek to find the BER when Tx1 and Tx2 use the same data rate. As described earlier, we consider an unequalized VPPM system. Assuming that the symbol detection method presented in Section II-D is used, the 


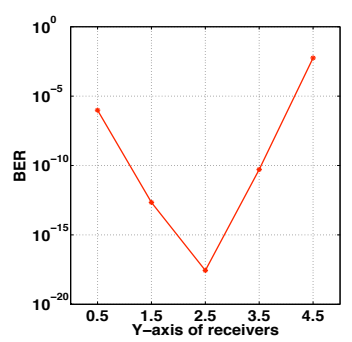

(a) BER vs Distance.

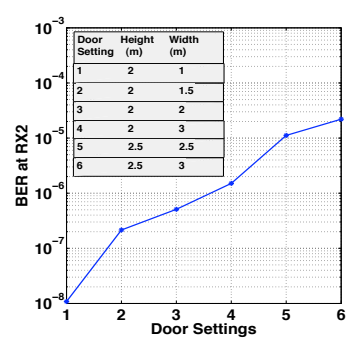

(b) BER at Rx2 vs Doorsizes.
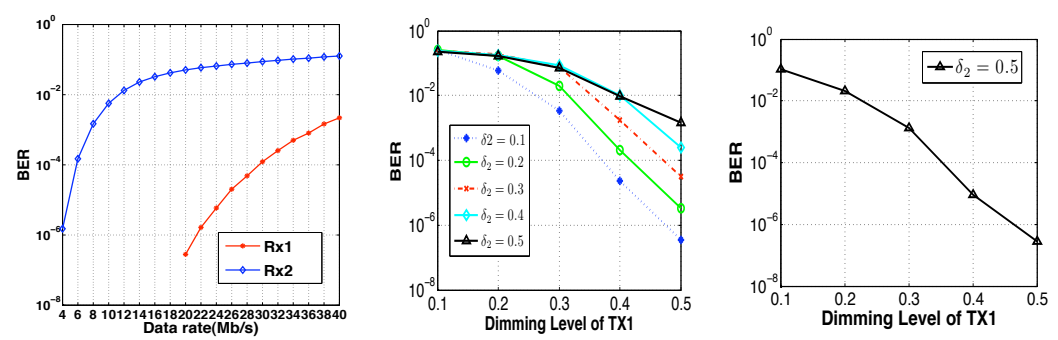

(d) BER vs Dimming at Rx2.

(e) BER vs Dimming at Rx1. and $\mathrm{R} \times 2$.

Fig. 6: BER for various parameters

probability of a bit error for the first scenario where both emitters transmit, can be estimated as:

$$
\begin{aligned}
P\{\text { bit error } & \left.\mid\left\{s_{1, i}, s_{2, j}\right\}\right\} \\
& \approx Q\left(\sqrt{\operatorname{SNR}_{\left\{s_{1, i}, s_{2, j}\right\}}}\right)
\end{aligned}
$$

where $s_{1, i}$ and $s_{2, j}$ are the symbols sent by $\mathrm{Tx} 1$ and $\mathrm{Tx} 2$ respectively, and $Q(x)$ is given by :

$$
Q(x)=\frac{1}{\sqrt{2 \pi}} \int_{x}^{\infty} \mathrm{e}^{-u^{2} / 2} d u
$$

For a random input data, the BER can be obtained by averaging over all possible symbols $s_{1, i}$ and over all possible interfering symbols $s_{2, j}$ from Tx2:

$$
\mathrm{BER}=\sum P\left\{\text { bit error } \mid\left\{s_{1, i}, s_{2, j}\right\}\right\} \cdot P\left\{\left\{s_{1, i}, s_{2, j}\right\}\right\}
$$

where $\left\{s_{1, i}, s_{2, j}\right\} \in S^{(1)}$ and $P\left\{\left\{s_{1, i}, s_{2, j}\right\}\right\}=1 / 4$.

\section{B. BER Performance}

We evaluate the BER performance with various combinations of the system parameters such as receiver positions, door sizes, data rates and dimming levels. If not stated otherwise, the two emitters are using the same dimming level and the same data rate.

BER vs Distance: Fig. 5(a) shows that when both emitters are transmitting, the receivers closer to the door are affected more. We calculate the BER for five receivers in Room 1, that are at different distances from the emitters. The two emitters use the same dimming level of 0.5 and a data rate of $10 \mathrm{Mbps}$. The BER results shown in Fig. 6(a) are consistent with the SNR results in Fig. 5(a).

BER vs Door Size: Our previous analysis and results have shown that the interfering signal from $\mathrm{Tx} 2$ impacts the performance in Room 1. It is interesting to look into how the different door sizes affects the performance. In Fig. 6(b) we show the BER at receiver RX2 for various door sizes. As expected, the larger the area of the door, the higher the BER. This is so because there is a higher likelihood that the interfering signal goes through the door when the door is larger.

BER vs Data Rate: Up to this point, we assume that the data rates of Tx1 and Tx2 are the same. In Fig. 6(c), we show the BER for Rx2 and Rx1 with different data rates. We increase the data rate by decreasing the pulse duration to fit in more pulses within a slot. Due to the interference at Rx2, the maximum data rate in order to achieve a minimum BER requirement of $10^{-6}$ is $4 \mathrm{Mbps}$. For Rx1, the corresponding maximum data rate is $20 \mathrm{Mbps}$. In general, increasing the data rate results in an increase of the BER at both receivers.

BER vs Dimming: In the previous sections, we consider the case where Tx1 and Tx2 both use dimming level of 0.5. Since dimming is a special feature of VPPM modulation, we look into the impact of dimming on the performance of the system. Let $\delta_{1}$ and $\delta_{2}$ be the dimming levels of Tx 1 and Tx2, respectively. The receivers at different locations in Room 1 are affected in different ways by the interfering signal from Tx2; thus, we look at the BER performance at $\mathrm{Rx} 1$ and $\mathrm{Rx} 2$ separately. We set the data rate of the connection to Rx1 to be $20 \mathrm{Mbps}$ and of the connection to Rx2 to be $4 \mathrm{Mbps}$.

Results shown in Fig. 6(d) present the BER performance at $\mathrm{Rx} 2$ for different values of $\delta_{1}$ and $\delta_{2}$. There are five sets of data, wherein for each the dimming level $\delta_{2}$ of Tx 2 is fixed and the dimming level $\delta_{1}$ of Tx 1 is increased from 0.1 to 0.5 . When $\delta_{2}$ is fixed, increasing $\delta_{1}$ improves the BER performance. For example, when $\delta_{2}$ is 0.2 , increasing $\delta_{1}$ from 0.3 to 0.5 makes the BER drop from $10^{-2}$ to $10^{-6}$. This trend is observable for each data set where $\delta_{2}$ is fixed at different levels. Also, if we look at Fig. 6(d) from a different angle, i.e. considering each column as a set of data, we can conclude that if $\delta_{1}$ is fixed, decreasing $\delta_{2}$ can improve the BER performance. Another observation is that when $\delta_{2}$ has a high value, for example if $\delta_{2}=0.5$, then tuning $\delta_{1}$ does not help improving BER. This is because the higher the value of $\delta_{2}$, the stronger the interfering signal, which impacts the BER performance. When $\delta_{1}=\delta_{2}=0.5$ the interfering signal for $\mathrm{Rx} 2$ is considerable.

As for Rx1, we observe that increasing $\delta_{1}$ improves the BER performance at Rx1. In Fig. 6(e) we show the results when $\delta_{2}=0.5$ and $\delta_{1}$ increases from 0.1 to 0.5 . We observe that increasing $\delta_{2}$ does not affect the BER performance at Rx1. This is so because Rx1 is close to Tx1 and far from the door so the interfering signal from $\mathrm{Tx} 2$ has limited impact on $\mathrm{Rx} 1$.

How to improve BER when dimming is limited: The previous results imply that increasing the dimming level of the desired emitter can improve BER performance. On the other hand, it also shown that when dimming reaches its limitation the BER cannot be improved much. For example, in Fig. 6(d) we see that if $\delta_{1}=\delta_{2}=0.5$, the BER at $\mathrm{Rx} 2$ is $10^{-3}$, a value that is not acceptable for practical settings. Thus, when there are constraints on the dimming level, we can reduce the data rate of Tx1 to improve the performance in Room 1. 

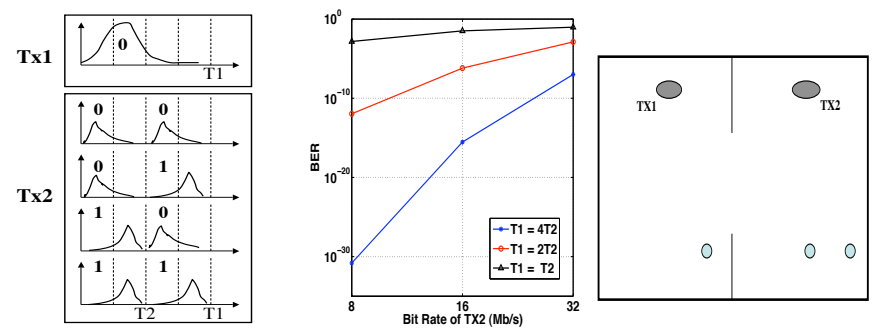

(a) Symbol set for $\mathrm{Tx} 1$ (b) BER at Rx2 for Tx1 (c) Receivers settings. transmitting $0, T_{1}=2 T_{2}$ and $\mathrm{Tx} 2$ using different data rates.

Fig. 7: Improving BER when dimming is limited.

The BER analysis in this case differs than the analysis in Section III-A. This happens because the possible combinations of symbols from Tx 1 and Tx2 are not the same as those in $S^{(1)}$ when $\mathrm{Tx} 1$ and $\mathrm{Tx} 2$ use the same data rate. In the following, we assume the data flows from Tx1 and Tx2 start at the same time to ease the analysis. If the data rate $R_{b_{1}}$ is half of $R_{b_{2}}$, the combination of symbols $\left\{s_{1, i}^{\prime}, s_{2, j}^{\prime}\right\}$ from Tx1 and Tx2 takes values in the set

$$
\begin{aligned}
S^{(2)}=\{ & \{0,00\},\{0,01\},\{0,11\},\{0,10\}, \\
& \{1,00\},\{1,01\},\{1,11\},\{1,10\}\}
\end{aligned}
$$

Because $R_{b_{1}}=\frac{1}{2} R_{b_{2}}$, it means $T_{1}=2 T_{2}\left(T_{1}, T_{2}\right.$ are the symbol duration times for Tx 1 and Tx 2 , respectively). Fig. 7(a) illustrates the symbol set for Tx1 transmitting 0 . The BER can be calculated using (13) and $P\left\{\left\{s_{1, i}, s_{2, j}\right\}\right\}$ is $1 / 8$ here.

Similarly, we can compute the BER for $T_{1}=4 T_{2}$. Fig. 7(b) shows the BER at RX2 when Tx1 and Tx 2 use different data rates. It is shown that increasing $T_{1}$ to be four times of $T_{2}$, i.e., reducing the data rate $\left(R_{b_{1}}\right)$ of Tx1 to be $1 / 4$ of the data rate $\left(R_{b_{2}}\right)$ of $\mathrm{Tx} 2$, can improve the BER performance at $\mathrm{Rx} 2$ significantly. Specifically, when the data rate $\left(R_{b_{2}}\right)$ of Tx2 is $16 \mathrm{Mbps}$, reducing $R_{b_{1}}$ from $16 \mathrm{Mbps}$ to $8 \mathrm{Mbps}$, drops the BER from $10^{-3}$ to $10^{-6}$. Moreover, if we reduce $R_{b_{1}}$ to $4 \mathrm{Mbps}$, the BER is as low as $10^{-16}$. Since the minimum required BER is $10^{-6}$, we set the maximum data rate of Tx1 accordingly, considering performance at $\mathrm{Rx} 2$ : for $R_{b_{2}}=32$ Mbps the maximum $R_{b_{1}}$ is $8 \mathrm{Mbps}$ and for $R_{b_{2}}=16 \mathrm{Mbps}$ the maximum $R_{b_{2}}$ is $8 \mathrm{Mbps}$. However note that the transmission of Tx 1 can adversely affect the receivers in Room 2. If the receiver is close to Tx2 or away from the door the impact is not as much, as shown in Fig. 7(c). The data rate of Tx1 can only be tuned to a lower value only if it does not affect the receiver in the other room.

\section{COnClusions}

In this paper, we discuss the performance of a visible light system within two neighboring rooms, where two emitters, Tx1 and Tx2, are located in separate rooms and VPPM with dimming is used. We propose an algorithm to characterize the channel impulse response and the BER of the system. Our results show that if $\mathrm{Tx} 2$, which is the interferer, is just illuminating, it does not impact the performance of the communication between $\mathrm{Tx} 1$ and the receivers in the same room. However, if both $\mathrm{Tx} 1$ and $\mathrm{Tx} 2$ are transmitting, the performance is degraded, especially for the receivers closer to the door. We show that increasing the dimming level of the desired signal can improve the BER performance. Moreover, we find that when the interfering signal is strong and the dimming level reaches its limit, reducing the data rate of $T x 1$ improves significantly the performance of the communication between Tx1 and the receivers in the same room; however, care must be taken when applying this strategy. We believe that our findings can help in designing MAC protocols for interference management with VLC.

\section{REFERENCES}

[1] T. Komine and M. Nakagawa, "Fundamental analysis for visible-light communication system using led lights," Consumer Electronics, IEEE Transactions on, vol. 50, no. 1, pp. 100-107, Feb.

[2] "Ieee standard for local and metropolitan area networks-part 15.7: Short-range wireless optical communication using visible light," IEEE Std 802.15.7-2011, pp. 1-309, 6.

[3] K. Cui, G. Chen, Z. Xu, and R. Roberts, "Line-of-sight visible light communication system design and demonstration," in Communication Systems Networks and Digital Signal Processing (CSNDSP), 2010 7th International Symposium on, July, pp. 621-625.

[4] D. O"Brien, L. Zeng, H. Le-Minh, G. Faulkner, J. Walewski, and S. Randel, "Visible light communications: Challenges and possibilities," in Personal, Indoor and Mobile Radio Communications, 2008. PIMRC 2008. IEEE 19th International Symposium on, Sept., pp. 1-5.

[5] J. Kahn and J. Barry, "Wireless infrared communications," Proceedings of the IEEE, vol. 85, no. 2, pp. 265-298, Feb.

[6] K. Lee, H. Park, and J. Barry, "Indoor channel characteristics for visible light communications," Communications Letters, IEEE, vol. 15, no. 2, pp. 217-219, February.

[7] F. J. Lopez-Hernandez, R. Perez-Jimenez, and A. Santamara, "Raytracing algorithms for fast calculation of the channel impulse response on diffuse ir wireless indoor channels," Optical Engineering, vol. 39, no. 10 , pp. 2775-2780, 2000.

[8] J. Barry, J. Kahn, W. Krause, E. Lee, and D. Messerschmitt, "Simulation of multipath impulse response for indoor wireless optical channels," Selected Areas in Communications, IEEE Journal on, vol. 11, no. 3, pp. 367-379, Apr.

[9] F. Lopez-Hernandez, R. Perez-Jimeniz, and A. Santamaria, "Monte carlo calculation of impulse response on diffuse ir wireless indoor channels," Electronics Letters, vol. 34, no. 12, pp. 1260-1262, Jun.

[10] F. Lopez-Hernandez, R. Perez-Jimenez, and A. Santamara, "Ray-tracing algorithms for fast calculation of the channel impulse response on diffuse ir wireless indoor channels," Optical Engineering, vol. 39, no. 10 , pp. 2775-2780, 2000.

[11] O. Gonzalez, S. Rodriguez, R. Perez-Jimenez, B. Mendoza, and A. Ayala, "Error analysis of the simulated impulse response on indoor wireless optical channels using a monte carlo-based ray-tracing algorithm," Communications, IEEE Transactions on, vol. 53, no. 1, pp. 124-130, Jan.

[12] M. Audeh, J. Kahn, and J. Barry, "Performance of pulse-position modulation on measured non-directed indoor infrared channels," Communications, IEEE Transactions on, vol. 44, no. 6, pp. 654-659, Jun.

[13] J. Barry, "Sequence detection and equalization for pulse-position modulation," in Communications, 1994. ICC '94, SUPERCOMM/ICC '94, Conference Record, 'Serving Humanity Through Communications.' IEEE International Conference on, May, pp. 1561-1565 vol.3.

[14] G. Forney, "Maximum-likelihood sequence estimation of digital sequences in the presence of intersymbol interference," Information Theory, IEEE Transactions on, vol. 18, no. 3, pp. 363-378, May.

[15] L. Zeng, D. O"brien, H. Le-Minh, K. Lee, D. Jung, and Y. Oh, "Improvement of date rate by using equalization in an indoor visible light communication system," in Circuits and Systems for Communications, 2008. ICCSC 2008. 4th IEEE International Conference on, May, pp. 678-682. 